\title{
BOUNDEDNESS AND COMPLETENESS IN LOCALLY CONVEX SPACES AND ALGEBRAS
}

\author{
GERARD A. JOSEPH \\ (Received 29 March, 1976) \\ Communicated by E. Strzelecki
}

\begin{abstract}
The set of bounded elements of a unital l.m.c. algebra is characterised as the union of certain naturally defined normed subalgebras, and an analogous characterisation is given for algebras of quotient-bounded operators on a locally convex space. Pseudocomplete l.m.c. algebras are characterised in terms of the completeness of these subalgebras, and analogously for operator algebras. Normcomplete locally convex spaces are characterised similarly, and the equivalence of this condition with the pseudocompleteness of the quotient-bounded operator algebras established. The scalar multiples of the identity in a unital l.m.c. algebra are characterised in terms of certain boundedness conditions.
\end{abstract}

\section{Introduction}

The central concept of this paper is that of a bounded element of a locally convex algebra, introduced by Allan (1965) in setting up a spectral theory for that class of algebras. An element is said to be bounded if some scalar multiple of it generates a bounded semigroup. It turns out (Theorem 2.4) that for a unital I.m.c. algebra $A$, the notion of boundedness of an element $a$ can be approached via a boundedness condition of the form

$$
\sup _{p \in P} p(\vec{a})<\infty,
$$

where $P$ is a calibration for $A$ satisfying certain conditions. This approach is the thrust of $\S 2$. For appropriate calibrations $P$ the above formula defines a unital subalgebra $A_{P}$ and the left-hand side an algebra norm on $A_{P}$. The algebras $A_{P}$ make their appearance many times in this paper.

In $\$ 3$ we consider the special case of I.m.c. operator algebras on a locally convex space. With each calibration $P$ for a locally convex space we associate a unital l.m.c. algebra $\mathscr{Q}_{P}$ of quotient-bounded operators (such operators 
appear in Moore (1968) as ultracontinuous operators). Theorem 3.6 is analogous to Theorem 2.4, and characterises the bounded elements of $\mathscr{Q}_{P}$ in terms of the normed subalgebras $\mathscr{B}_{p}$ of universally bounded operators, which correspond to the algebras $A_{P}$ above.

Allan (1965) has given the notion of pseudocompleteness of a locally convex algebra. For a unital l.m.c. algebra $A$, this condition has a simple interpretation in terms of the completeness of the normed subalgebras $A_{P}$ (Theorem 4.7). Moore (1969) has given the similar notion of normcompleteness of a locally convex space, which permits a characterisation similar to that of pseudocompleteness (Theorem 4.5). In fact Moore has shown that normcompleteness is equivalent to completeness of all the algebras $\mathscr{B}_{P}$ of universally bounded operators, and using this result we can relate normcompleteness to pseudocompleteness of the algebras $\mathscr{Q}_{P}$ of quotient-bounded operators (Theorem 4.9).

$\$ 5$ is a collection of results whose theme is the characterisation of the scalar multiples of the identity in a unital l.m.c. algebra. It is shown that elements which are not scalar multiples of the identity are 'unbounded' in various senses. Some of the results apply to normed spaces. For example, Corollary 5.8 asserts that a bounded linear operator on a normed space, which is not a scalar multiple of the identity, has arbitrarily large operator norms corresponding to equivalent norms on the underlying space.

The material of this paper forms part of the author's doctoral thesis (Joseph (1975)) submitted at the University of Newcastle, N.S.W. The author gratefully acknowledges the award of a research scholarship from that university. He also expresses his sincere thanks to Professor J. R. Giles under whose supervision the research was carried out.

\section{Preliminaries}

All locally convex spaces will be assumed Hausdorff and over the complex field C. A calibration for a locally convex space $E$ is any family $P$ of seminorms generating its topology, in the sense that the topology of $E$ is the coarsest with respect to which all the seminorms in $P$ are continuous. A calibration $P$ is characterised by the property that the sets

$$
\{x \in E: p(x) \leqq \varepsilon\}, \quad(\varepsilon>0, p \in P)
$$

constitute a neighborhood sub-base at 0 . Because of the Hausdorff assumption, every calibration $P$ is separating: if $p(x)=0$ for every $p \in P$, then $x=0$. The set of calibrations for $E$ is denoted $\mathscr{P}(E)$. The family of all continuous seminorms on $E$ is denoted $\Lambda(E)$ and is the largest member of $\mathscr{P}(E)$. The identity operator is denoted $I$, and the dual of $E$ is denoted $E^{*}$. 
A locally convex algebra is an algebra with a locally convex topology in which multiplication is separately continuous. Such an algebra is locally $m$-convex (l.m.c.) if it has a neighborhood base $\mathcal{U}$ at 0 such that each $U \in \mathscr{U}$ is convex, balanced $(\lambda U \subseteq U$ for $|\lambda| \leqq 1)$, and satisfies the semigroup property $U^{2} \subseteq U$. Any algebra with identity will be called unital. A unital l.m.c. algebra $A$ is characterised by the existence of a calibration $P$ such that each $p \in P$ is submultiplicative $(p(x y) \leqq p(x) p(y))$ and satisfies $p(1)=1$. The set of such calibrations is denoted $\mathscr{P}^{*}(A)$. The family of all continuous submultiplicative seminorms $p$ on $A$ satisfying $p(1)=1$ is denoted $\Lambda^{*}(A)$, and is the largest member of $\mathscr{P}^{*}(A)$. The term unital normed algebra implies that the norm is submultiplicative and satisfies $\|1\|=1$.

Any family of seminorms on a linear space $X$ is partially ordered by the relation $\leqq$, where $p \leqq q$ means $p(x) \leqq q(x)$ for all $x \in X$. Any such family is preordered by the relation $\leqslant$, where $p \leqslant q$ means there exists $r>0$ such that $p(x) \leqq r q(x)$ for all $x \in X$. If $p \leqslant q$ and $q \leqslant p$, we write $p \simeq q$, and this defines an equivalence relation on any family of seminorms on $X$. We use this relation to define an equivalence relation between families of seminorms.

Definition 1.1. Families $P_{1}, P_{2}$ of seminorms on a linear space are called $Q$-equivalent (denoted $P_{1}=P_{2}$ ) provided

(i) for each $p_{1} \in P_{1}$ there exists $p_{2} \in P_{2}$ such that $p_{1} \simeq p_{2}$; and

(ii) for each $p_{2} \in P_{2}$ there exists $p_{1} \in P_{1}$ such that $p_{1} \simeq p_{2}$.

$Q$-equivalent, separating families on a linear space generate the same locally convex topology. We will also require the following stronger equivalence relation.

Definition 1.2. Families $P_{1}, P_{2}$ of seminorms on a linear space are called $B$-equivalent (denoted $P_{1} \sim P_{2}$ ) provided each seminorm in each is a positive multiple of a seminorm in the other.

A calibration $P \in \mathscr{P}(E)$ will be called basic provided the sets

$$
\{x \in E: p(x) \leqq \varepsilon\}, \quad(p \in P, \varepsilon>0)
$$

constitute a neighborhood base at 0 . We can relate the property of being basic to the preorder $\leqslant$ defined above.

Proposition 1.3. A calibration $P$ is basic if and only if it is upwards directed by $\leqslant ;$ (that is, for each $p_{1}, p_{2} \in P$ there exist $p \in P$ such that $\left.p_{1} \leqslant p, p_{2} \leq p\right)$.

A calibration $Q$-equivalent to a basic calibration is basic.

Finally we note that if $p$ is a seminorm on a linear space $X$ and $T$ a linear operator on $X$, then the composition $p T$, where $p T(x)=p(T x)$, is a seminorm 
on $X$; if $f$ is a linear functional on $X$, then $|f|$ is a seminorm on $X$, where $|f|(x)=|f(x)|$.

\section{The bounded elements of a unital l.m.c. algebra}

We give the definition of a bounded element, and show that in a unital l.m.c. algebra, boundedness of an element is equivalent to another kind of boundedness condition. Recall that a set $B$ in a linear topological space is bounded if it is absorbed by every neighborhood of 0 , which in a locally convex space $E$ amounts to the condition

$\sup \{p(x): x \in B\}<\propto$ for all $p$ belonging to some calibration $P \in \mathscr{P}(E)$.

DEFINITION 2.1. An element $a$ of a locally convex algebra $A$ is bounded if for some $r>0$, the set $\left\{\left(r^{-1} a\right)^{n}: n=1,2, \cdots\right\}$ is bounded. Thus $a$ is bounded if for some $r>0, r^{-1} a$ generates a bounded semigroup.

The set of bounded elements of $A$ is denoted $A_{0}$.

Definition 2.2. The radius of boundedness of an elelment $a \in A_{0}$ is the number

$$
\beta(a)=\inf \left\{r>0: r^{-1} a \text { generates a bounded semigroup }\right\} .
$$

For the remainder of this section, $A$ denotes a unital I.m.c. algebra. We adopt the abbreviations $\mathscr{P}^{*}$ and $\Lambda^{*}$ for $\mathscr{P}^{*}(A)$ and $\Lambda^{*}(A)$ respectively. Given $P \in \mathscr{P}^{*}$, we define a subset $A_{P}$ of $A$ by the condition

$$
\sup _{p \in P} p(a)<\infty
$$

it is clear that $A_{P}$ is a unital subalgebra of $A$. For $a \in A_{P}$ we define

$$
\|a\|_{P}=\sup _{p \in P} p(a)
$$

then $\left(A_{P},\|\cdot\|_{P}\right)$ is a unital normed algebra, whose $\|\cdot\|_{P}$-topology is finer than that inherited from $A$. Our characterisation of $A_{0}$ (Theorem 2.4) depends on the following recalibration lemma, a direct generalisation of the classical renorming theorem for normed algebras (see for example Bonsall and Duncan (1971), p. 21).

Lemma 2.3. Let $S$ be a bounded semigroup in $A$, and $\mathscr{H}$ any $Q$ equivalence class of calibrations in $\mathscr{P}^{*}$. Then there exists $P \in \mathscr{H}$ such that $S$ lies within the unit ball of $\left(A_{P},\|\cdot\|_{P}\right)$.

Proof. Choose any $P \in \mathscr{H}$ and suppose without loss of generality that $1 \in S$. For each $p \in P$ define $p^{\prime}$ on $A$ by 


$$
p^{\prime}(a)=\sup \{p(s a): s \in S\} .
$$

Then $p^{\prime}$ is a continuous submultiplicative seminorm on $A, p^{\prime} \simeq p$, and $p^{\prime}(s a) \leqq p^{\prime}(a)$ for each $s \in S$ and $a \in A$. Now define $p^{\prime \prime}$ on $A$ by

$$
p^{\prime \prime}(b)=\sup \left\{p^{\prime}(b x): p^{\prime}(x) \leqq 1\right\} .
$$

Then $p^{\prime}$ is a continuous submultiplicative seminorm on $A, p^{\prime} \simeq p$, and $p^{\prime}(s a) \leqq p^{\prime}(a)$ for each $s \in S$ and $a \in A$. Now define $p^{\prime \prime}$ on $A$ by

$$
p^{\prime \prime}(b)=\sup \left\{p^{\prime}(b x): p^{\prime}(x) \leqq 1\right\} .
$$

Then $p^{\prime \prime} \in \Lambda^{*}$ and $p^{\prime \prime} \simeq p$, so $P^{\prime \prime}=\left\{p^{\prime \prime}: p \in P\right\} \simeq P$, hence $P^{\prime \prime} \in \mathscr{H}$; further, $p^{\prime \prime}(s) \leqq 1$ for all $s \in S$ and $p \in P$.

THeOREM 2.4. Let $\mathscr{H}$ be any $Q$-equivalence class in $\mathscr{P}^{*}$. An element of $A$ is bounded if and only if it belongs to $A_{P}$ for some $P \in \mathscr{H}$; thus

$$
A_{0}=\bigcup_{P \in \mathscr{K}} A_{P}
$$

Proof. Let $a \in A_{0}$ and $r>0$ such that $r^{-1} a$ generates a bounded semigroup. By Lemma 2.3 there exists $P \in \mathscr{H}$ such that $\left\{\left(r^{-1} a\right)^{n}: n=1,2, \cdots\right\}$ lies within the unit ball of $\left(A_{P},\|\cdot\|_{P}\right)$, which implies that $p(a) \leqq r$ for all $p \in P$, so $a \in A_{P}$ and $\|a\|_{P} \leqq r$. Conversely, if $a \in A_{P}$ for some $P \in \mathscr{H}$, it is clear that $\|a\|_{p}^{-1} a$ generates a bounded semigroup.

From the proof of Theorem 2.4 we deduce immediately a formula for the radius of boundedness of a bounded element.

Corollary 2.5. For each $a \in A_{0}$,

$$
\beta(a)=\inf \left\{\|a\|_{P}: P \in \mathscr{H}, a \in A_{P}\right\} .
$$

Note. For each $P \in \mathscr{H}$, the unit ball of $A_{P}$ is a closed, convex, balanced, bounded semigroup in $A$, and by Lemma 2.3 every such semigroup lies in the unit ball of $A_{P}$ for some $P \in \mathscr{H}$. Thus Theorem 2.4 follows also from a result of Allan (1965), p. 401.

ExAmple 2.6. Let $A=s$ be the unital l.m.c. algebra of all complex sequences $x=\left(x_{1}, x_{2}, \cdots\right)$ with the topology of pointwise convergence. Let $P$ be the calibration $\left\{p_{1}, p_{2}, \cdots\right\}$ defined by $p_{n}(x)=\left|x_{n}\right|$. Then $A_{P}$ is the algebra $\ell^{x}$ of bounded sequences and $\|\cdot\|_{P}$ is the usual supremum norm.

\section{Bounded elements of operator algebras}

Here we turn attention to certain I.m.c. algebras of operators on a locally convex space. Our main result here (Theorem 3.6) is essentially the operator analog of Theorem 2.4, and describes the bounded elements of the algebras of 
quotient-bounded operators in terms of the normed subalgebras of universally bounded operators. Throughout this section $E$ denotes a locally convex space; we write $\mathscr{P}$ for $\mathscr{P}(E)$.

The first class of operators to be defined appears in Moore (1969) and Chilana (1970).

Definition 3.1. A linear operator $T$ on $E$ is universally bounded (UB) with respect to a calibration $P \in \mathscr{P}$ if there exists $r>0$ such that $p T \leqq r p$ for all $p \in P$. The class of such operators is denoted $\mathscr{B}_{P}$. For $T \in \mathscr{B}_{P}$ we define

$$
\|T\|_{P}=\inf \{r>0: p T \leqq r p \text { for all } p \in P\} .
$$

The following properties of UB operators are obvious.

Proposition 3.2. (i) $\mathscr{B}_{P}$ is a unital subalgebra of the algebra of continuous linear operators on $E$;

(ii) $\left(\widehat{n}_{P},\|\cdot\|_{P}\right)$ is a unital normed algebra;

(iii) if $P^{\prime} \in \mathscr{P}$ such that $P^{\prime} \sim P$, then $\mathscr{B}_{P^{\prime}}=\mathscr{B}_{P}$ and $\|\cdot\|_{P^{\prime}}=\|\cdot\|_{P}$.

In the next section we consider questions relating to completeness of the algebras $\mathscr{B}_{p}$.

The following class of operators was first defined in Appendix A of Michael (1952); they appear also in Moore (1968) and Chilana (1972) and numerical range theory for operators of this class was studied in Giles, Joseph, Koehler and Sims (1975).

Definition 3.3. A linear operator $T$ on $E$ is quotient-bounded $(Q B)$ with respect to a calibration $P \in \mathscr{P}$ if $p T \leqslant p$ for each $p \in P$. The class of such operators is denoted $\mathscr{Q}_{P}$. For each $T \in \mathscr{Q}_{P}$ and $p \in P$ we define

$$
\hat{p}(T)=\inf \{r>0: p T \leqq r p\} .
$$

For each $p \in P, \hat{p}$ is then a submultiplicative seminorm on $\mathscr{Q}_{P}$ satisfying $\hat{p}(I)=1$, and we denote by $\hat{P}$ the family $\{\hat{p}: p \in P\}$. The following assertions are clear.

Proposition 3.4. (i) $\mathscr{Q}_{P}$ is a unital subalgebra of the algebra of continuous linear operators on $E$;

(ii) $\mathscr{Q}_{P}$ is a unital l.m.c. algebra with respect to the topology determined by $\hat{P}$, and $\hat{P} \in \mathscr{P}^{*}\left(\mathscr{Q}_{P}\right)$;

(iii) $\mathscr{B}_{P}$ is a unital subalgebra of $\mathscr{Q}_{P}$, and in fact $\mathscr{B}_{P}=\left(\mathscr{Q}_{P}\right)_{\hat{P}},\|\cdot\|_{P}=\|\cdot\|_{P}$;

(iv) if $P^{\prime} \in \mathscr{P}$ such that $P^{\prime} \simeq P$, then $\mathscr{Q}_{P}=\mathscr{Q}_{P^{\prime}}$ and $\hat{P} \simeq \hat{P}^{\prime}$; thus the $\hat{P}$ - and $\hat{P}^{\prime}$-topologies coincide;

(v) the $\hat{P}$-topology is finer than the topology of uniform convergence on the bounded subsets of $E$. 
For the remainder of this section, $P$ denotes a fixed calibration in $\mathscr{P}$. Again our main result requires a recalibration lemma; the following result is analogous to Lemma 2.3 and generalises the classical renorming theorem for operators on normed spaces (see for example Bonsall and Duncan (1971), p. 90).

Lemma 3.5. Let $\mathscr{S}$ be a bounded semigroup in $2_{p}$. Then there exists $P^{\prime} \in \mathscr{P}$ such that $P^{\prime} \simeq P$ and $\mathscr{S}$ lies within the unit ball of $\left(\mathscr{B}_{P},\|\cdot\|_{P^{\prime}}\right)$.

Proof. Suppose without loss of generality that $I \in \mathscr{S}$. For each $p \in P$ define $p^{\prime}$ on $E$ by

$$
p^{\prime}(x)=\sup \{p(T x): T \in \mathscr{S}\} .
$$

Then $p^{\prime}$ is a continuous seminorm on $E$ and $p^{\prime} \simeq p$. Thus $P^{\prime}=\left\{p^{\prime}: p \in P\right\} \in \mathscr{P}$ and $P^{\prime} \simeq P$. For each $T \in \mathscr{Y}$ it is then clear that $T \in \mathscr{B}_{P^{\prime}}$ and that $\|T\|_{P^{\prime}} \leqq 1$. We are now able to characterise the bounded elements of $\mathscr{Q}_{p}$ as those operators in $\mathscr{Q}_{P}$ which are UB with respect to some calibration $Q$-equivalent to $P$.

THEOREM 3.6. An operator $T \in \mathscr{Q}_{p}$ is a bounded element of $\mathscr{Q}_{p}$ if and only if there exists $P^{\prime} \in \mathscr{P}$ such that $P^{\prime} \simeq P$ and $T \in \mathscr{B}_{P^{\prime}} ;$ thus

$$
\left(\mathscr{Q}_{P}\right)_{0}=\cup\left\{\mathscr{B}_{P^{\prime}}: P^{\prime} \in \mathscr{P}, P^{\prime} \simeq P\right\} .
$$

Proof. The proof is similar to that of Theorem 2.4, but invokes Lemma 3.5 instead of Lemma 2.3.

Again the proof yields a formula for the radius of boundedness $\beta_{P}(\cdot)$ in $\mathscr{Q}_{p}$.

Corollary 3.7. For each $T \in\left(\mathscr{Q}_{\mathbf{P}}\right)_{0}$,

$$
\beta_{P}(T)=\inf \left\{\|T\|_{P^{\prime}}: P^{\prime} \in \mathscr{P}, P^{\prime} \simeq P, T \in \mathscr{B}_{P}\right\} .
$$

Note. By (iv) of Proposition 3.4, $Q$-equivalent recalibrations change neither the algebra of QB operators nor its topology. Theorem 3.6 gives an indication of the extent to which the algebras of UB operators may change under such recalibrations. A fuller account of their behavior is given in $\$ 5$.

\section{Completeness conditions}

In this section we consider two completeness conditions, normcompleteness (Moore (1969)), and its algebra analog, pseudocompleteness (Allan (1965)). We characterise normcomplete locally convex spaces in terms of the completeness of certain normed subspaces (Theorem 4.5), and pseudocom- 
plete l.m.c. algebras in terms of the completeness of corresponding normed subalgebras (Theorem 4.7), which are just the subalgebras $\boldsymbol{A}_{P}$ discussed in $\$ 2$. For the particular case of l.m.c. algebras of $\mathrm{QB}$ operators, we obtain the appropriate operator analog of Theorem 4.7 (Theorem 4.8), which then yields a connection between normcompleteness of a locally convex space and pseudocompleteness of its algebras of $\mathrm{QB}$ operators.

Let $E$ be a locally convex space. Denote by $\mathscr{A}$ the class of closed, convex, balanced, bounded (ccbb) subsets of $E$. For $B \in \mathscr{A}$ denote by $E_{B}$ the linear span of $B$ and define $\|\cdot\|_{B}$ on $E_{B}$ to be the Minkowski functional of $B$ :

$$
\|x\|_{B}=\inf \left\{r>0: r^{-1} x \in B\right\} .
$$

Proposition 4.1. For each $B \in \mathscr{A},\left(E_{B},\|\cdot\|_{B}\right)$ is a normed linear space with unit ball $B$, whose $\|\cdot\|_{B}$-topology is finer than that inherited from $E$.

DEFINITION 4.2. $E$ is normcomplete provided $\left(E_{B},\|\cdot\|_{B}\right)$ is complete for all $B \in \mathscr{A}$.

It is shown in Moore (1967) that normcompleteness is weaker than sequential completeness, and Moore (1969) has in fact proved the remarkable result that $E$ is normcomplete if and only if $\left(\mathscr{B}_{P},\|\cdot\|_{P}\right)$ is complete for all $P \in \mathscr{P}$. We now define a system of subspaces which occur among the $E_{B}, B \in \mathscr{A}$, whose completeness turns out to guarantee normcompleteness (Theorem 4.5).

Given a calibration $P \in \mathscr{P}$, the following condition (identical to (1) in $\$ 2$ )

$$
\sup _{p \in P} p(x)<\infty
$$

defines a linear subspace $E_{P}$ of $E$, and the formula

$$
\|x\|_{P}=\sup _{p \in P} p(x)
$$

defines a norm on $E_{P}$ which induces a topology finer than that inherited from $E$. We require two lemmas, the first of which is similar to Lemma 2.3.

Lemma 4.3. Let $B$ be a bounded set in $E$ and $\mathscr{H}$ any $B$-equivalence class of calibrations in $\mathscr{P}$. Then there exists $P \in \mathscr{H}$ such that $B$ lies within the unit ball of $\left(E_{P},\|\cdot\|_{P}\right)$.

Proof. Take any $P \in \mathscr{H}$; for each $p \in P$ let $k_{p}=\sup \{p(x): x \in B\}$, and define $p^{\prime}=p /\left(k_{p}+1\right)$; letting $P^{\prime}=\left\{p^{\prime}: p \in P\right\}$, we have $P^{\prime} \sim P$ so $P^{\prime} \in \mathscr{H}$, and clearly $B \subseteq E_{P}$ and $\|x\|_{P^{\prime}} \leqq 1$ for all $x \in B$.

A subcollection $\mathscr{U}_{0}$ of a collection $\mathscr{U}$ of sets is said to be cofinal in $\mathscr{U}$ if every set in $U$ is contained in a set in $\mathscr{U}_{0}$. The following lemma shows that to establish normcompleteness it is only necessary to consider a cofinal subcol- 
lection of $\mathscr{A}$. The proof uses the fact that a complete space is normcomplete, a consequence of the result of Moore (1967) referred to above.

Lemma 4.4. Let $\mathscr{A}_{0}$ be cofinal in $\mathscr{A}$, and suppose $\left(E_{B},\|\cdot\|_{B}\right)$ is complete for all $B \in \mathscr{A}_{0}$. Then $E$ is normcomplete.

Proof. Take any $B \in \mathscr{A}$, and let $B_{0} \in \mathscr{A}_{0}$ such that $B \subseteq B_{0}$. Since $B$ is closed in $E$, it is also closed in $E_{B_{1}}$ in the finer topology induced by its norm $\|\cdot\|_{B_{0}}$. Since $B_{0}$ is the unit ball of $\left(E_{B_{0}},\|\cdot\|_{B_{0}}\right), B$ is bounded in $E_{B_{0}}$. Thus $B$ is a ccbb set in $E_{B_{b}}$, which is complete by hypothesis and hence normcomplete. But this implies that $\left(E_{B},\|\cdot\|_{B}\right)$ is complete as required.

Our characterisation of normcompleteness now follows simply.

THEOREM 4.5. Let $\mathscr{H}$ be any $B$-equivalence class in $\mathscr{P}$. Then $E$ is normcomplete if and only if $\left(E_{P},\|\cdot\|_{P}\right)$ is complete for all $P \in \mathscr{H}$.

Proof. For each $P \in \mathscr{H},\left(E_{P},\|\cdot\|_{P}\right)$ is equal to $\left(E_{B},\|\cdot\|_{B}\right)$ where $B$ is the unit ball of $\left(E_{P},\|\cdot\|_{P}\right)$. Thus the 'only if' part is clear by definition of normcompleteness. By Lemma 4.3 , the unit balls of the $\left(E_{P},\|\cdot\|_{P}\right), P \in \mathscr{H}$, are cofinal in $\mathscr{A}$; the converse is then immediate from Lemma 4.4.

Allan's pseudocompleteness is defined similarly to normcompleteness, but pays regard to the multiplicative structure of a locally convex algebra, and is accordingly slightly weaker.

Definition 4.6. A locally convex algebra $A$ is pseudocomplete provided $\left(A_{B},\|\cdot\|_{B}\right)$ is complete for every ccbb semigroup $B$ in $A$.

Our characterisation of pseudocomplete l.m.c. algebras is analogous to Theorem 4.5. The proof follows that of Theorem 4.5, using Lemma 2.3 in place of Lemma 4.3, and is omitted.

THEOREM 4.7. Let $A$ be a unital l.m.c. algebra, and $\mathscr{H}$ any $Q$ equivalence class in $\mathscr{P}^{*}$. Then $A$ is pseudocomplete if and only if $\left(A_{P},\|\cdot\|_{P}\right)$ is complete for each $P \in \mathscr{H}$.

When $A$ is the algebra $\mathscr{Q}_{P}$ for some calibration $P$ for $E$, we can give an operator analog of Theorem 4.7.

THEOREM 4.8. Let $P \in \mathscr{P}$; then $\mathscr{Q}_{P}$ is pseudocomplete if and only if $\mathscr{B}_{P}$. is complete for all $P^{\prime} \in \mathscr{P}$ such that $P^{\prime} \simeq P$.

Proof. Lemma 3.5 implies that the unit balls of the $\left(\mathscr{B}_{P} \cdot\|\cdot\|_{P}\right), P^{\prime} \simeq P$, are a cofinal family of ccbb semigroups in $\mathscr{Q}_{p}$. The result then follows as in Theorems 4.5 and 4.7 .

Finally in this section we use a result of Moore, alluded to earlier, to link the two completeness conditions discussed here. 
THEOREM 4.9. E is normcomplete if and only if $\mathscr{Q}_{P}$ is pseudocomplete for all $P \in \mathscr{P}$.

Proof. By Theorem 4.8 the latter condition is equivalent to completeness of all the $\mathscr{B}_{P}, P \in \mathscr{P}$, which by Theorem 3 of Moore (1969) is equivalent to normcompleteness.

\section{Characterisations of the scalar multiples of the identity}

In a unital l.m.c. algebra $A$, a scalar multiple $a=\lambda 1$ of the identity is a bounded element, and also has the properties that $p(a)$ is constant for all $p \in \Lambda^{*}$, that $a \in A_{P}$ for all $P \in \mathscr{P}^{*}$, and that $\|a\|_{P}$ is constant for all $P \in \mathscr{P}^{*}$. The results of this section show that these properties in fact characterise the scalar multiples of the identity among all bounded elements. These results make essential use of the Hahn-Banach Theorem. Our discussion begins with the case of a unital l.m.c. algebra $A$, then turns as usual to the case of an algebra of $\mathrm{QB}$ operators on a locally convex space $E$.

Recall from $\$ 1$ the definition of a basic calibration. We denote by $\mathscr{P}_{b}$ the set of basic calibrations for $E$, and by $\mathscr{P}_{b}^{*}$ the set of basic calibrations for $A$ which belong to $\mathscr{P}^{*}$. We denote by $\langle x, y\rangle$ the linear span of vectors $x$ and $y$, and by $\mathrm{C} 1$ (or $\mathrm{CI}$ as appropriate) the one-dimensional subalgebra spanned by the identity.

The following lemma will often be requied in conjunction with the Hahn-Banach Theorem.

Lemma 5.1. Let $x, y \in E$ be linearly independent and let $P \in \mathscr{P}_{b}$. Then there exists $p \in P$ whose restriction to $\langle x, y\rangle$ is a norm.

Proof. Since $P$ is both separating and basic, we can choose $p_{1} \in P$ such that $p_{1}(x) \neq 0, p_{1}(y) \neq 0$. If the restriction of $p_{1}$ to $\langle x, y\rangle$ is not already a norm, its null-space is one-dimensional, spanned by some $z \in\langle x, y\rangle$, and there exists $p_{2} \in P$ such that $p_{2}(z) \neq 0$. Choosing $p \in P$ such that $p_{1} \leq p$ and $p_{2} \leq p$, we have the result.

Our first group of results concerns a unital l.m.c. algebra $A$.

Proposition 5.2. Let $a \in A$ such that $a \notin \mathrm{C} 1$. Then for any $P \in \mathscr{P}_{b}^{*}$, the set of numbers

$$
\left\{p^{\prime}(a): p^{\prime} \in \Lambda^{*}, p^{\prime} \simeq p \text { for some } p \in P\right\}
$$

is unbounded.

Proof. Choose $p \in P$ whose restriction to $\langle a, 1\rangle$ is a norm, and let $R>0$ be arbitrary. By the Hahn-Banach Theorem there exists $f \in A^{*}$ such 
that $f(a)=R, f(1)=0$, and $|f| \leqslant p$. Define $p_{1}=\max (p,|f| t)$. Then $p_{1}$ is a continuous seminorm on $A$ and $p_{1} \simeq p$. Define $p_{2}$ on $A$ by

$$
p_{2}(b)=\sup \left\{p_{1}(b x): p_{1}(x) \leqq 1\right\}
$$

Then $p_{2} \in \Lambda^{*}, p_{2} \simeq p$, and $p_{2}(a) \geqq p_{1}(a) \geqq R$, which yields the result.

When $A$ is normable (admits a calibration $\{\|\cdot\|\}$ ), denote by $N^{*}$ the set of norms in $\Lambda^{*}$ which define the topology of $A$.

Corollary 5.3. Let $A$ be normable, and $a \in A$ such that $a \notin C 1$. Then $\left\{\|a\|:\|\cdot\| \in N^{*}\right\}$ is unbounded.

Proof. Choose any $\|\cdot\|_{0} \in N^{*}$ and set $P=\left\{\|\cdot\|_{0}\right\}$ in Proposition 5.2. Recall from Theorem 2.4 that the set $A_{0}$ of bounded elements is precisely the union of all the $A_{P}, P \in \mathscr{H}$, where $\mathscr{H}$ is any $Q$-equivalence class in $\mathscr{P} *$. The next result shows that for bounded elements $a$ which are not scalar multiples of the identity, the values of $\|a\|_{P}$, for $P \in \mathscr{H}$ such that $a \in A_{P}$, are arbitrarily large.

Corollary 5.4. Let $a \in A_{0}$ such that $a \notin C 1$, and let $\mathscr{H}$ be any $Q$ equivalence class in $\mathscr{P}_{b}^{*}$. Then

$$
\left\{\|a\|_{P}: P \in \mathscr{H}, a \in A_{P}\right\}
$$

is unbounded.

Proof. Let $R>0$ be arbitrary, and let $P \in \mathscr{H}$ such that $a \in A_{p}$. By Proposition 5.2 there exists $p^{\prime} \in \Lambda^{*}$ such that $p^{\prime} \simeq p$ for some $p \in P$, and $p^{\prime}(a) \geqq R$. Then $P^{\prime}=P \cup\left\{p^{\prime}\right\} \in \mathscr{H}, a \in A_{p}$, and $\|a\|_{r} \geq R$.

Corollary 5.5. Let $\mathscr{H}$ be any $Q$-equivalence class in $\mathscr{P}_{h}^{*}$. Then

$$
\bigcap_{P \in H} A_{P}=\mathrm{Cl}
$$

Proof. Let $a \in A$, such that $a \notin \mathrm{C} 1$, and let $P \in \mathscr{K}$ such that $a \in A_{P}$. By Proposition 5.2 there exist $p \in P$ and $p_{1}, p_{3}, \cdots \in \Lambda^{*}$ such that $p_{n} \simeq p$ and $p_{n}(a) \geqq n$ for each $n$. Then $P^{\prime}=P \cup\left\{p_{1}, p_{2}, \cdots\right\} \in \mathscr{H}$ and $a \notin A_{P}$.

Corollany 5.6. For each $P \in \mathscr{P}_{b}^{*}$ there exists $P_{0} \in \mathscr{P}_{b}^{*}$ such that $P_{0} \simeq P$ and $A_{P_{11}}=\mathrm{Cl}$.

ProOf. Let $\mathscr{H}$ be the $Q$-equivalence class in $\mathscr{P}_{h}^{*}$ containing $P$, and let $P_{0}=\cup \mathscr{H}$. Then $P_{0} \simeq P$ and

$$
A_{P_{0}} \subseteq \bigcap_{P^{\prime} \in \mu^{\prime}} A_{p^{\prime}}=\mathrm{C} 1
$$


Our second group of results concerns the l.m.c. algebras $\mathscr{L}_{p}$ of $\mathrm{QB}$ operators on a locally convex space $E$, and follows a pattern analogous to that of the preceding results for a general unital l.m.c. algebra. If $T$ is a linear operator on $E$ and $p \in \Lambda=\Lambda(E)$ such that $p T \leqslant p$, we write

$$
\begin{aligned}
\hat{p}(T) & =\inf \{r>0: p T \leqq r p\} \\
& =\sup \{p(T x): p(x) \leqq 1\} .
\end{aligned}
$$

Proposition 5.7. Let $P \in \mathscr{P}_{b}$ and $T \in \mathscr{Q}_{P}$ such that $T \notin \mathrm{CI}$. Then the set of numbers

$$
\left\{\hat{p}(T): p \in \Lambda, p \simeq p_{1} \text { for some } p_{1} \in P\right\}
$$

is unbounded.

Proof. Let $R>0$ be arbitrary. We distinguish two cases.

Case 1. For some $x, y \neq 0$ we have $T x=\lambda x, T y=\mu y$ where $\lambda, \mu \in \mathrm{C}, \lambda \neq \mu$. By Lemma 5.1 there exists $p_{1} \in P$ whose restriction to $\langle x, y\rangle$ is a norm, and we may suppose $p_{1}(x) \leqq \frac{1}{2}, p_{1}(y) \leqq \frac{1}{2}$. By the Hahn-Banach Theorem there exists $f \in E^{*}$ such that $f(x)=f(y)=R|\lambda-\mu|^{-1}$ and $|f| \leqslant p_{1}$. Defining $p=\max \left(p_{1},|f|\right)$, we have $p \approx p_{1}, p(x-y) \leqq 1$, and $p(T(x-y)) \geqq$ $|f(\lambda x-\mu y)|=R$, so $\hat{p}(T) \geqq R$.

Case 2. For some $x \in E, x$ and $T x$ are linearly independent. Choose $p_{1} \in P$ whose restriction to $\langle x, T x\rangle$ is a norm; then we may suppose $p_{1}(x) \leqq 1$. The Hahn-Banach Theorem gives $f \in E^{*}$ such that $f(x)=0, f(T x)=R$, and $|f| \leqq p_{1}$. Defining $p=\max \left(p_{1},|f|\right)$, we have $p(x) \leqq 1, p(T x) \geqq R$, so $\hat{p}(T) \geqq R$.

When $E$ is normable, denote by $N$ the set of norms in $\Lambda$ which define the topology of $E$.

COROllary 5.8. Let E be normable, $T$ a bounded linear operator on $E$ such that $T \notin C I$. Then $\{\|T\|:\|\cdot\| \in N\}$ is unbounded.

Proof. Choose any $\|\cdot\|_{0} \in N$ and set $P=\left\{\|\cdot\|_{0}\right\}$ in Proposition 5.7. Recall from Theorem 3.6 that for $P \in \mathscr{P}$, the bounded elements of $\mathscr{Q}_{\boldsymbol{P}}$ are precisely those operators in $\mathscr{Q}_{P}$ which belong to $\mathscr{B}_{P}$, for some $P^{\prime} \in \mathscr{P}$ such that $P^{\prime} \simeq P$. For operators $T \in\left(\mathscr{Q}_{P}\right)_{11}$ which do not belong to $C I$, the values of $\|T\|_{P^{\prime}}$, for $P^{\prime}=P$ such that $T \in \mathscr{B}_{P^{\prime}}$, are arbitrarily large.

Corollary 5.9. Let $P \in \mathscr{P}_{b}$ and $T \in\left(\mathscr{Q}_{\mathrm{P}}\right)_{0}$ such that $T \notin \mathrm{C} I$. Then

is unbounded.

$$
\left\{\|T\|_{P^{\prime}}: P^{\prime} \in \mathscr{P}_{b}, P^{\prime} \simeq P, T \in \mathscr{B}_{P^{\prime}}\right\}
$$


Proof. Let $R>0$ be arbitrary, and let $P_{1} \in \mathscr{P}_{b}$ such that $P_{1} \simeq P$ and $T \in \mathscr{B}_{P_{1}}$. By Proposition 5.7 there exists $p \in \Lambda$ such that $p \simeq p_{1}$ for some $p_{1} \in P_{1}$ and $\hat{p}(T) \geqq R$. Then $P^{\prime}=P_{1} \cup\{p\} \simeq P, T \in \mathscr{B}_{P^{\prime}}$, and $\|T\|_{P^{\prime}} \geqq R$.

Corollary 5.10 . Let $\mathscr{H}$ be any $Q$-equivalence class in $\mathscr{P}_{b}$. Then

$$
\bigcap_{P \in \mathscr{K}} \mathscr{B}_{P}=\mathrm{C} I \text {. }
$$

Proof. Let $P \in \mathscr{H}$ and let $T \in\left(\mathscr{Q}_{P}\right)_{0}$ such that $T \notin \mathrm{CI}$. By Proposition 5.7 there exist $p \in P$ and $p_{1}, p_{2}, \cdots \in \Lambda$ such that $p_{n} \simeq p$ and $\hat{p}_{n}(T) \geqq n$ for each $n$. Then $P^{\prime}=P \cup\left\{p_{1}, p_{2}, \cdots\right\} \in \mathscr{H}$ and $T \notin \mathscr{B}_{P^{\prime}}$.

Corollary 5.11. For each $P \in \mathscr{P}_{b}$ there exists $P_{0} \in \mathscr{P}_{b}$ such that $P_{0} \simeq P$ (so that $\mathscr{Q}_{P_{0}}=\mathscr{Q}_{P}$ ) but $\mathscr{B}_{P_{0}}=\mathrm{CI}$.

ProOf. Let $\mathscr{H}$ be the $Q$-equivalence class in $\mathscr{P}_{b}$ containing $P$, and let $P_{0}=\cup \mathscr{H}$. Then $P_{0} \simeq P$ and

$$
\mathscr{B}_{P_{0}} \subseteq \bigcap_{P^{\prime} \in \mathscr{H}} \mathscr{B}_{P^{\prime}}=C I
$$

The preceding results demonstrate that 'wild' behavior of the algebras of UB operators under $Q$-equivalent recalibrations of $E$, in contrast to the algebras of QB operators, which remain unchanged under such recalibrations. It follows from the last two results that the scalar multiples of the identity are the only operators which are UB with respect to every calibration for $E$. Our final result shows that such operators are the only ones which are in fact QB with respect to every calibration for $E$.

Proposition 5.12. Let $P \in \mathscr{P}$ satisfy $\left\{|f|: f \in E^{*}\right\} \subseteq P$. Then $\mathscr{Q}_{P}=\mathrm{CI}$.

Proof. Suppose $T \notin C I$. As in Proposition 5.7 we distinguish two cases.

Case 1. For some $x, y \neq 0$ we have $T x=\lambda x, T y=\mu y$ where $\lambda, \mu \in \mathrm{C}, \lambda \neq \mu$. Choose $f \in E^{*}$ such that $f(x)=f(y)=1$ and define $p=|f|$. Then $p \in P, p(x-y)=0$, but $p(T(x-y))=|\lambda-\mu| \neq 0$, so clearly $T \notin \mathscr{Q}_{p}$.

Case 2. For some $x \in E, x$ and $T x$ are linearly independent. Choose $f \in E^{*}$ such that $f(x)=0, f(T x)=1$, and define $p=|f|$. Then $p(x)=0$ but $p(T x)=1$ so again $T \notin \mathscr{Q}_{P}$.

\section{References}

G. R. Allan (1965), 'A spectral theory for locally convex algebras', Proc. London Math. Soc. (3) 15. 399-421.

F. F. Bonsall and J. Duncan (1971), Numerical ranges of operators on normed spaces and of elements of normed algebras (London Math. Soc. Lecture Note Series, 2. Cambridge). 
A. K. Chilana (1970). 'Invariant subspaces for linear operators in locally convex spaces'. $J$. London Math. Soc. (2) 2, 493-503.

A. K. Chilana (1972), 'Some special operators and new classes of locally convex spaces', Proc. Cambridge Philos. Soc. 71, 475-490.

J. R. Giles, G. Joseph, D. O. Koehler and B. Sims (1975), 'On numerical ranges of operators on locally convex spaces', J. Austral. Math. Soc. 20, 468-482.

G. A. Joseph (1975). 'Numerical range and operators on locally convex spaces'. Ph.D. Thesis, University of Newcastle. N.S.W.

E. A. Michael (1952), 'Locally multiplicatively-convex topological algebras', Mem. Amer. Math. Soc. 11.

R. T. Moore (1967). 'Completeness, equicontinuity and hypocontinuity in operator algebras', $J$. Functional Analysis 1, 419-442.

R. T. Moore (1968). Measurable. continuous and smooth vectors for semigroups and group representations', Mem. Amer. Math. Soc. 78.

R. T. Moore (1969), 'Banach algebras of operators on locally convex spaces'. Bull. Amer. Math. Soc. $75.68-73$.

\section{Department of Mathematics,}

University of Newcastle,

New South Wales, 2308,

Australia. 